and the uric acid diathesis were the chief sources of error.

The inadequacy of puncturing the organ, and even its thorough palpation as final tests of the presence or absence of stone may be shown by the experience of Morris. The coterie of so-called stone symptoms was typical in a case in charge of this capable surgeon. Exploration through a lumbar cut, needling and renal incision brought negative results! The patient survived the operation. The symptoms recurred and we are compelled to admire the courage of Dr. Morris who removed the suspected kidney by the lumbar route. He could feel with the fingers no induration in its substance, when after nephrectomy the specimen lay before him. After incising it at several points he finally found, however, a stone of some size embedded in the renal tissue. The patient got well. Dr. Howard Kelley's recent article in the News will probably teach us much by the use of instruments of precision; for catherization of the ureters, perfected as he states, will show us at least the kind of work done by either kidney, The patient, Mr. A., æt. 49, a merchant from Newark, Ohio, was brought to the writer by Dr. Notring, of that place. He had been an invalid for three years. His appearance was that of a man accustomed to great suffering; he walked in such a way as to favor the left side; he was badly stooped, and the concavity of the bend of his body was to the front and left. His side having been exposed, he was given a sharp punch in the lame loin, the thumb being forced in the direction of the kidney. $\mathrm{He}$ complaimed that he felt as if a sword had been stuck into him. Jordan Lloyd looks upon a statement of this kind by the patient, after receiving such a thrust, as being strongly suggestive of the presence of renal calculus. A uranalysis at the time elicited the following facts: Sp. gr., 1012; reaction acid; phosphates present; no casts.

About forty attacks of renal colic had occurred within the three years of invalidism preceding his visit. Each meant a week's illness in bed. Pain began commonly in the night, starting in the left loin. A so-called swelling showed itself in that region during these seizures and disappeared after them. More or less complete anuria accompanied this intumescence, and was followed by a copious discharge of urine each time. Increasing intensity of this loin-ache with frequent and painful dribbling acts of urination, were invariable accompaniments of these paroxysms. The free administration of morphia was necessary during them. Neither pus nor blood nor sabulous matter in the urine was ever observed by the patient or the attending physician. To make things more obscure, in a violent lifting effort, made about the time that his illness began, a left inguinal hernia was produced. He naturally attributed much of his discomfort to this fact. $\mathrm{He}$ was told that he had a stone in the left kidney. An operation was performed at his home on Aug. 4., 1895. The usual preparation was employed. Strychnia was given beforehand in thirtieth grain doses for a few days, for its stimulant and tonic effect; while the parts were cleansed and suitably prepared to minimize the risk of sepsis; ether narcosis; right lateral position; a hard pillow was placed beneath the right loin, so that the left costo-iliac space was thus put on the stretch and increased. A four-inch oblique incision was made, avoiding the pleura above and peritoneum to the ventral side. The kidney was readily exposed; the finger was passed about the organ to separate the fatty capsule. On palpating its posterior surface toward the hilum a hard mass could be felt in the pelvis, which it well-nigh filled. Invaluable aid was rendered by the careful management of the anesthetic by Dr. Notring and by the strong upward traction of the ribs by Dr. Spear, so that the kidney could be brought well into the parietal opening. A probe was carried into the more accessible lower segment of the cortex, radially toward the pelvis, while the organ was steadied by the thumb and fingers. A stone was felt. The instrument in place served as a guide to the guarded bistoury with which the wound was enlarged; while a pair of angular Wells' forceps facilitated extraction of the stone. A drain was inserted into the loin; no vessels were ligated. Some shock supervened, but disappeared, however, within three hours. A few silk stitches were inserted. Some small sabulous fragments were discharged through the natural passages in the next few days. For a fortnight, urine was somewhat bloody, and afterward became cloudy.

Profiting by a previous experience in which after crushing a vesical calculus of the uric acid variety, the frequent voiding of cloudy urine had been a source of anxiety to the patient, the very free drinking of lithia water was advised and the urine became clear. He had quite recovered within a month so as to be able to pursue his occupation. The stone, a uric acid concretion, weighed $73.5 \mathrm{grs}$. It occupied the pelvis of the kidney which it almost filled, while the upper part of it lay embedded in the renal tissue. It might be well in closing to allude to the striking and misleading facts of the case, viz.: the absence of pus and blood and calcareous matter in the urine. $\mathrm{He}$ was at times, no doubt, a sufferer from acute hydro-nephrosis, as his history indicated.

\section{CLINICAL LECTURE.}

Extract from of a Clinical Lecture Delivered before the Senior Students at the Wisconsin College of Physicians and Surgeons.

BY JAMES A. BACH, M.D.

PROFESSOR OF OPHTHALMOLOGY AND OTOLOGY. MILWAUKEE, WIS.

Gentremen:-Our first patient to-day is one of unusual interest. This young lady, as she tells us, about three years ago had the misfortune to receive a slight injury of her right eye, which injury was followed by deep infection, producing a plastic iridocyclitis. In due course of time the fellow eye became affected with a sympathetic inflammation, which practically amounted to an extension of the infection through the optic nerve sheaths, leading to simjlar results, i.e., a plastic inflammation of the uveal tract with extensive adhesions. The inflammatory process in both eyes has now come to a standstill, with the result that the girl has simply a perception of light left.

Covering one eye and moving this light across different parts of the visual field you will notice that she readily locates the light correctly; in other words, her light projection is good, showing that the perceptive organs are, to say the least, partially intact in every portion of the field. The readiness with which she locates this light, although only a very small amount can pass into the interior of her eye, owing to these dense plastic exudates in the pupillary field, gives us a reasonable assurance that if we can suc- 
cessfully remove these dense obstructions to the light we may gain some very serviceable vision for this young lady. She has everything to gain and practically nothing to lose.

Before attempting to do this operation I should like to eall your attention to some instructive features in this case. In the first place, by looking closely at the eyes with the assistance of oblique illumination you will observe that the anterior chamber is perfectly absent; the iris lying immediately behind the cornea conforming itself perfectly to the curvature of the cornea. A condition similar to this is of ten found after a neglected iritis with complete posterior synechiæ, only that in these cases the iris does not adapt itself to the cornea, but bulges forward in the middle and is held down at the pupillary margins by adhesions, producing what is known as iris bombé. This bulging is produced by the filling in of aqueous humor in the posterior chamber, where it is chiefly secreted, which, however, can not find its way forward into the anterior chamber owing to the pupillary adhesions cutting off the communication between the chambers. In this case before you there is no bulging present, because there is no aqueous humor in the posterior chamber, the organs for its production having evidently been destroyed during the process of this deep and severe inflammation and adhesions, which is not the case when the inflammation is con. fined, or chiefly so, to the iris alone. These eyes evidently have lost their aqueous humor and with it the power to reproduce the same. As a rule, eyes of this kind experience a decided fluctuation in their tension. At about the time of the inflammation, owing to insufficiency of elimination of the internal fluids, the tension may rise very materially and may become $\mathbf{T}=+2$. The use of atropin in this stage is often not permissible, owing to this tendency. After the subsidence of this inflammation, the insufficiency of internal secretion of fluid, together with a gradual degeneration and shrinking of the contents of the globe, produced by the cutting off of the proper nutrition to these non-vascular structures, will gradually allow a decline in tension which often goes so far as to result in atrophy of the eyeball, known as phthisis bulbi. The tension of these eyes is as yet but very slightly reduced. If you will again look at the eyes under illumination you will observe the pupillary field to be very small, and instead of black as it ordinarly is, it is a yellowish white. This is a dense plastic exudate that has been thrown out during the process of inflammation and has become organized. But very little light can penetrate it. The iris looks slightly stretched and thinned; it has, however, not lost as much of its luster as one often finds in these cases.

We will now thoroughly cocainize this eye and proceed to make a large iridectomy. We will, no doubt, find that the whole of the iris has become adherent to the lens so that after the iridectomy the endothelial pigment layer of the iris will remain adherent to the anterior capsule of the lens, necessitating the removal of the lens as well. These lenses, owing to the insufficient nutrition are cataractous, as a rule. As I now enter my knife at the limbus and penetrate the cornea, if you will watch closely, you will observe that it at once impinges upon the iris, but by careful manipulation I succeed, as you see, to make my corneal section, although too small for our purpose. I will now take these delicate scissors and on either side enlarge my incision to sufficiency. As I grasp the iris with my forceps, I can feel distinctly its adherence to the lens capsule, and drawing a portion of it out it is snipped off. As anticipated, the pigment layer still rests on the lens. I now pass the cystotome into the anterior chamber and tear the capsule of the lens, which being completed the lens presents itself and is delivered without any difficulty. As you see, the patient is overjoyed at the fact of again receiving sufficient light to perceive objects. A similar case was operated at this clinic last year with a very satisfactory result. A fact that makes this case of special interest is that she has been refused operative interference from a number of oculists and she will indeed have occasion to feel grateful should the results prove such as we now have every reason to believe that they will be. It is very important in this class of cases not to operate while there are still active inflammatory symptoms present, because you are very liable to again lose through further plastic exudates the pupillary freedom gained by the operation.

Case 2.-This old gentleman, while a soldier in our late war, contracted trachoma, and has ever since been troubled with this unpleasant disease, not having received any special treatment for the same. Not only are his lids deeply infiltrated with the products of this disease, but it seems, on close examination, that the iris and other internal structures of the eye ball have suffered in consequence. He now comes to us with double senile cataract, which are mature. Under these conditions it always is a grave undertaking to open the eye ball, especially where the interference of the internal delicate structures is as great as becomes necessary during the removal of a cataract. The chances for infection become a serious consideration. Although he has been under treatment for his granulations for some time now, vet the conjunctival sac is still not in a sufficiently healthy condition to justify subjecting this man to the operation of extraction. In these cases it is a good plan and one that I always pursue, to divide the operation into two parts and in that manner lessen very materially the chances for infection. By making a preliminary iridectomy to.day only, the chances for infection are not great and in the course of a few weeks, having received more treatment for the trachoma, we can with reasonable safety remove the lens. Any unhealthy condition of the conjunctival sac where this can be done ought to always be remedied before making the operation for cataract, otherwise we may expect from time to time to lose eyes from infections. I believe it is chiefly owing to the great care I take in preparing my cataract patients that $I$ have not sustained a single loss of an eye through infection after cataract extraction; my success has been uniform.

Privilege not Waived.--In the case of Barker v. Cunard Steamship Co., decided by the general term of the supreme court of New York, Dec. 18, 1895, the court holds that it was not error to refuse to permit the defendant to prove by a certain physician the condition of the plaintiff when he entered a hospital, and the statements made by him in respect to the previous condition of his health. The knowledge of this witness was acquired from an inspection of and conversation with the plaintiff while the relation of physician and patient existed between them, and was privileged. This privilege, the court declares, was not waived by the plaintiff's having called other physicians to testify to his condition anterior and subsequent to the time he was in the hospital. 